\title{
Soft Handover Overhead Reduction by RAKE Reception with Finger Replacement*
}

\author{
Seyeong Choi \\ Dept. of Electrical \& Computer Eng. \\ Texas A\&M University \\ College Station, TX 77843, USA \\ Email: yeong@ece.tamu.edu
}

\author{
Mohamed-Slim Alouini, Khalid A. Qaraqe \\ Dept. of Electrical Eng. \\ Texas A\&M University at Qatar \\ Education City, Doha, Qatar \\ Email: \{alouini, khalid.qaraqe\}@qatar.tamu.edu
}

\author{
Hong-Chuan Yang \\ Dept. of Electrical \& Computer Eng. \\ University of Victoria \\ BC, V8W 3P6, Canada \\ Email: hyang@ece.uvic.ca
}

\begin{abstract}
We propose and analyze a new finger replacement technique that is applicable for RAKE receivers when they operate in the soft handover (SHO) region. In the SHO region, the receiver uses by default the strongest paths from the serving base station (BS) and only when the combined signal-to-noise ratio falls below a certain pre-determined threshold, the receiver uses more resolvable paths from the target $\mathrm{BS}$ to improve the performance. Instead of changing the configuration for all fingers, the receiver just compares the sum of the weakest paths from the serving BS with that of other paths from the target BS and select the better group. Applying some recent results on order statistics, we attack the statistics of two partial sums of order statistics and provide an analytical framework for the performance evaluation of the proposed scheme. By investigating the tradeoff between the error performance and the SHO overhead, we show through numerical examples that the new scheme can save some SHO overhead load.
\end{abstract}

\section{INTRODUCTION}

To mitigate the effect of multi-path fading, RAKE receivers are used in conjunction with wideband code division multiple access (WCDMA) systems. These receivers combine the resolvable paths in order to increase the overall signal-to-noise ratio (SNR) and lower the probability of deep fades [1, Section 9.5.1]. In the handover $(\mathrm{HO})$ region, the number of available resolvable paths can be quite high since they come from the serving base station (BS) and the target BS. As such, it is of interest to develop diversity combining schemes that maintain low complexity and low processing power consumption for mobile units while using a minimal amount of additional network resources.

Although many low-complexity diversity combining schemes have been proposed and studied over the last decade [2]-[12], they still result in a significant increase in network overhead (known as a soft HO (SHO) overhead) if they are used without any modification. Recently, the authors proposed and analyzed the performance of a new finger combining scheme that maintain a low complexity and reduce the SHO overhead [13]. The main idea behind [13] is that, in the SHO region whenever the received signal is unsatisfactory, the receiver scans the additional resolvable paths from the target BS and selects the strongest paths among the total available paths from both the serving and the target BS.

\footnotetext{
* This work was supported in part by the Qatar Foundation for Education, Science, and Community Development, Qatar, in part by Qatar Telecom (Qtel), Qatar, and in part by a Discovery Grant from NSERC, Canada.
}

In this paper we propose an alternative finger combining scheme that is also applicable to the SHO region and which further reduces the SHO overhead at the expense of a certain degradation in performance (that we will accurately quantify in the body of our paper). With this scheme, we assume that the $L_{c}$ out of total $L$ resolvable paths from the serving BS are by default assigned to the RAKE fingers of the mobile unit in the SHO region following $L_{c} / L$-generalized selection combining (GSC) type of combining. Only when the output SNR falls below a predetermined fixed SNR threshold (known also as a target SNR), the receiver asks for the additional resources from the target BS. More specifically, the receiver scans the additional $L_{a}\left(\leq L_{c}\right)$ resolvable paths from the target BS, compares the sum of the SNRs of $L_{a}$ paths from the target BS with the sum of the $L_{a}$ weakest SNRs among $L$ paths from the serving BS, and selects the better group to form $L_{c}$ paths again. Unlike the scheme proposed in [13], the scheme proposed and studied in this paper just compares two sums and as such avoids reconfiguring all fingers in the SHO region.

The main contribution of this paper is to provide an analytical framework deriving the statistics of the receiver output SNR of our newly proposed scheme, including its cumulative density function (CDF) and probability density function (PDF). In our derivations, we specifically tackle the joint PDF of two adjacent partial sums of order statistics. These results are then used to analyze the performance in terms of the average probability of error and to investigate the tradeoff between complexity and performance by quantifying the SHO overhead versus the target SNR. To simplify our analysis and make it tractable, we assume that the receiver operates over a "perfect" uniform propagation delay profile provided by a multi-path searcher in a way that the multi-path components are correctly assigned to the RAKE fingers.

The remaining of this paper is organized as follows. In Section II, we present the system and channel model under consideration as well as the mode of operation of the proposed scheme. Based on this mode of operation, we derive in Section III the statistics of the combined SNR in the form of finite integrations of elementary functions. These results are next applied in Section IV to the performance analysis of the proposed system. This section also illustrates the tradeoff of complexity versus performance by considering the SHO overhead of our proposed scheme. Finally, Section V provides some concluding remarks. 


\section{Finger RePlacement-BASED RAKE COMBINING}

\section{A. Channel and System Model}

Let $\gamma_{i}$ denote the instantaneous received SNR of the $i$ th resolvable path, $i=1,2, \cdots, L+L_{a}$. We assume that the signals from all the resolvable paths experience independent and identically distributed (i.i.d.) Rayleigh fading environments. Under a block fading assumption, the fading channel gain of each path is assumed to be constant over one time slot and vary independently from one slot to the next. As such, the faded SNR, $\gamma_{i}$, follows the same exponential distribution, with common PDF and CDF given as [1, Eq. (6.5)]

$$
f_{\gamma}(x)=\frac{1}{\bar{\gamma}} \exp \left(-\frac{x}{\bar{\gamma}}\right), \quad x \geq 0
$$

and

$$
F_{\gamma}(x)=1-\exp \left(-\frac{x}{\bar{\gamma}}\right), \quad x \geq 0,
$$

respectively, where $\bar{\gamma}$ is the common average faded SNR.

Next, we consider systems that employ a RAKE receiver. More specifically, we assume that, in the SHO region, there are $L$ available resolvable paths from the serving $\mathrm{BS}$ and $L_{a}$ additional available paths from the target BS, and depending on the channel conditions only $L_{c}$ paths among $L$ or $L+$ $L_{a}\left(L_{a} \leq L_{c} \leq L\right)$ paths are used for RAKE reception according to the mode of operation described in the next section.

\section{B. Mode of Operation}

Without loss of generality, we assume that at first the receiver relies only on $L$ resolvable paths gathered from the serving BS and as such starts with $L_{c} / L$-GSC. At the beginning of every time slot, the receiver compares the received SNR, $\Gamma_{L_{c}: L}$, with a certain target SNR, denoted by $\gamma_{T}$, where $\Gamma_{i: j}$ is the sum of the $i$ largest SNRs among $j$ ones, i.e., $\Gamma_{i: j}=\sum_{k=1}^{i} \gamma_{k: j}$ where $\gamma_{k: j}$ is the $k$ th order statistics (see [4] for terminology). If $\Gamma_{L_{c}: L}$ is greater than or equal to $\gamma_{T}$, a oneway $\mathrm{SHO}^{1}$ is used and no finger replacement is needed. On the other hand, whenever $\Gamma_{L_{c}: L}$ falls below $\gamma_{T}$, a two-way $\mathrm{SHO}^{2}$ is attempted. In this case, the RAKE receiver compares the sums of two groups, the sum of the currently connected $L_{a}$ smallest paths, $\sum_{i=L_{c}-L_{a}+1}^{L_{c}} \gamma_{i: L}$, from the serving BS and the sum of the $L_{a}$ additional paths, $\sum_{i=1}^{L_{a}} \gamma_{i}$, from the target BS. Then, the receiver replaces the $L_{a}$ smallest paths which are currently used with the best group. Note that no replacement occurs if the sum of the $L_{a}$ additional paths from the target BS is less than the sum of the $L_{a}$ weakest paths from the serving BS.

For simplicity, if we let

$$
Y=\sum_{i=1}^{L_{c}-L_{a}} \gamma_{i: L}
$$

\footnotetext{
${ }^{1}$ One-way SHO refers to the scenario in which the mobile unit is connected only to the serving BS while being in the SHO region.

${ }^{2}$ Two-way SHO refers to the scenario in which the mobile unit is connected to the serving and the target BSs while being in the SHO region.
}

$$
Z=\sum_{i=L_{c}-L_{a}+1}^{L_{c}} \gamma_{i: L}
$$

and

$$
W=\sum_{i=L+1}^{L+L_{a}} \gamma_{i}
$$

then, based on the above mode of operation, the final combined $\mathrm{SNR}, \gamma_{t}$, is given by

$$
\gamma_{t}= \begin{cases}Y+\max \{Z, W\}, & 0 \leq Y+Z<\gamma_{T} \\ Y+Z, & Y+Z \geq \gamma_{T} .\end{cases}
$$

\section{Statistics of The Combined SNR}

In this section, we derive the statistics of the combined SNR of the proposed scheme. In order to do that, we need to consider the joint PDF of two adjacent partial sums of order statistics. Hence, first we provide an analytical framework for the joint PDF of two partial sums of order statistics which is later on used for the statistics of the combined SNR.

Because of space limitations, we only present in this paper some of the final results. The specific details behind the derivations can be found in the journal version [14].

\section{A. $C D F$}

From (6), the CDF of $\gamma_{t}, F_{\gamma_{t}}(x)$, can be written as

$$
\begin{aligned}
& F_{\gamma_{t}}(x) \\
& =\left\{\begin{array}{l}
\operatorname{Pr}[Y+\max \{Z, W\}<x], \quad 0 \leq x<\gamma_{T} ; \\
\operatorname{Pr}\left[\gamma_{T} \leq Y+Z<x\right] \\
+\operatorname{Pr}\left[Y+\max \{Z, W\}<x, Y+Z<\gamma_{T}\right], x \geq \gamma_{T} .
\end{array}\right.
\end{aligned}
$$

If we let $l=L_{c}-L_{a}$ and $k=L_{c}$, then the order statistics of $L$ resolvable paths can be viewed as

$$
\underbrace{\overbrace{\gamma_{1: L}, \cdots, \gamma_{l-1: L}}^{A=\sum_{i=1}^{l-1} \gamma_{i: L}}, \gamma_{l: L}}_{Y=\sum_{i=1}^{l} \gamma_{i: L}}, \overbrace{Z=\sum_{i=l+1}^{k} \gamma_{i: L}}^{\overbrace{\gamma_{l+1: L}, \cdots, \gamma_{k-1: L}}^{B=\sum_{i=l+1}^{k-1} \gamma_{i: L}}, \gamma_{k: L}}, \cdots, \gamma_{L: L} .
$$

We can obtain a closed-form expression for the joint PDF, $f_{A, \gamma_{l: L}, B, \gamma_{k: L}}(a, \alpha, b, \beta)$, for i.i.d. Rayleigh fading channels as

$$
\begin{aligned}
& f_{A, \gamma_{l: L}, B, \gamma_{k: L}}(a, \alpha, b, \beta) \\
& =\frac{L ! e^{-(a+\alpha+b+\beta) / \bar{\gamma}}\left(1-e^{-\beta / \bar{\gamma}}\right)^{L-k}[a-(l-1) \alpha]^{l-2}}{(L-k) !(k-l-1) !(k-l-2) !(l-1) !(l-2) ! \bar{\gamma}^{k}} \\
& \quad \times \sum_{j=0}^{k-l-1}\left(\begin{array}{c}
k-l-1 \\
j
\end{array}\right)(-1)^{j}[b-\beta(k-l-j-1) \\
& -\alpha j]^{k-l-2} \mathcal{U}(\alpha) \mathcal{U}(\alpha-\beta) \mathcal{U}(a-(l-1) \alpha) \\
& \quad \times \mathcal{U}(b-\beta(k-l-j-1)-\alpha j), \\
& \quad 0<(k-l-1) \beta<b<(k-l-1) \alpha .
\end{aligned}
$$

Since $Y=A+\gamma_{l: L}$ and $Z=B+\gamma_{k: L}$, we can obtain the joint pdf of $Y$ and $Z, f_{Y, Z}(y, z)$, from (9) by integrating out 
$\gamma_{l: L}$ and $\gamma_{k: L}$ as

$$
\begin{aligned}
& f_{Y, Z}(y, z) \\
& =\int_{0}^{\frac{z}{k-l}} \int_{\frac{z}{k-l}}^{\frac{y}{l}} f_{A, \gamma_{l: L}, B, \gamma_{k: L}}(y-\alpha, \alpha, z-\beta, \beta) d \alpha d \beta \\
& \quad y>\frac{l}{k-l} z .
\end{aligned}
$$

Note that (10) involves only finite integrations of elementary functions. Therefore, even though a closed-form expression is tedious to obtain, this joint probability can be easily calculated with mathematical software, such as Mathematica.

\section{B. $P D F$}

With the help of Leibnitz's rule [15, Eq. (6.40)] and the differentiation (7) with respect to $x$, we can obtain after some manipulations the following generic expression for the PDF of the combined SNR, $\gamma_{t}$, as

$$
\begin{aligned}
& f_{\gamma_{t}}(x) \\
& = \begin{cases}\int_{0}^{x}\left(f_{Y, Z}(y, x-y) F_{W}(x-y)\right. \\
\left.+f_{W}(x-y) \int_{0}^{x-y} f_{Y, Z}(y, z) d z\right) d y, & 0 \leq x<\gamma_{T} \\
f_{Y+Z}(x)+\int_{0}^{\gamma_{T}}\left(f_{W}(x-y)\right. & \\
\left.\times \int_{0}^{\gamma_{T}-y} f_{Y, Z}(y, z) d z\right) d y, & x \geq \gamma_{T},\end{cases}
\end{aligned}
$$

where $f_{Y, Z}(\cdot, \cdot)$ is defined in $(10), F_{W}(\cdot)$ and $f_{W}(\cdot)$ are the CDF and the PDF of $L_{a}$-fold MRC output SNR [1, Eqs. $(6.25)(6.23)]$, respectively, and $f_{Y+Z}(\cdot)$ is the PDF of $L_{c} / L-$ GSC [16, Eq. (9.433)].

\section{NUMERICAL EXAMPLES}

In this section, we apply the results from the previous section to the performance analysis of our proposed combining scheme over i.i.d. Rayleigh fading channels. More specifically, by presenting some selected numerical examples, we first examine its average bit error rate (BER). We then look into the SHO overhead it requires.

\section{A. Average BER Comparison}

The average bit error rate can be calculated by finding the expected value of the conditional probability of error.

In Fig. 1, we plot the average BER of BPSK versus the average SNR per path, $\bar{\gamma}$, of the proposed scheme for various values of $L_{c}$ over i.i.d. Rayleigh fading channels when $L=$ $5, L_{a}=1$, and $\gamma_{T}=5 \mathrm{~dB}$. It is clear from this figure that better performance can be obtained by increasing the number of fingers at the receiver. Also we can observe the typical diminishing returns effect of diversity systems, i.e., the improvement in performance is decreasing as $L_{c}$ increases.

Next, we consider in Figs. 2 and 3 the effect of the switching threshold on the performance. Fig. 2 represents the average BER of BPSK versus the average SNR per path, $\bar{\gamma}$, of the proposed scheme for various values of $\gamma_{T}$ over i.i.d. Rayleigh fading channels when $L=5, L_{c}=3$, and $L_{a}=2$. From this figure, it is clear that the higher the threshold, the better the performance, as we expect intuitively. However, in good channel conditions (i.e., $\bar{\gamma}$ is relatively large compared to
$\gamma_{T}$ ), our scheme becomes insensitive to variations in $\gamma_{T}$. This is because when $\bar{\gamma}$ becomes larger, the combined SNR is typically large enough in a way that it does not need to rely on the additional paths from the target BS. For a better understanding of this issue, we vary in Fig. 3 the number of replacement paths, $L_{a}$, with two values of $\gamma_{T}$. When the threshold is set high, the performance improves as $L_{a}$ increases while in the case of the low threshold the increase of the number of replacement paths, $L_{a}$, does not improve the performance since in this case no replacement is needed.

We now compare our scheme with the alternative replacement scheme which was considered in the companion paper [13]. For convenience sake, we call our scheme as a block change scheme and the scheme in [13] as a full GSC scheme. Recall that in the full GSC scheme, instead of the block change, the largest $L_{c}$ paths are selected among $L+L_{a}$ ones. It is clear that the full GSC is expected to provide better performance than the block change scheme. However, in the block change scheme considered in this paper, we can reduce the load induced by a full reordering process of all the paths at a slight of sacrifice in performance. Note that both schemes require the same number of path estimations.

In Fig. 4, we show the average BER of BPSK versus the average SNR per path, $\bar{\gamma}$, of the block change scheme and the full GSC scheme for the different values of $\gamma_{T}$ over i.i.d. Rayleigh fading channels when $L=4, L_{c}=3$, and $L_{a}=2$. When the switching threshold is small, both schemes have almost the same performance since the additional paths are not necessary. On the other hand, in the case of large threshold values, the full GSC scheme shows better performance, as expected. For the better illustration of the comparison, in Fig. 5 we plot the average BER of BPSK versus the normalized output threshold, $\gamma_{T} / \bar{\gamma}$, of the block change scheme and the full GSC scheme for different values of $L_{a}$ over i.i.d. Rayleigh fading channels when $L=4, L_{c}=3$, and $\bar{\gamma}=0 \mathrm{~dB}$. As we can see, the performance improves as the ratio of the switching threshold to the average SNR per path increases. Note that both schemes shows the same performance when $L_{a}=1$, while the full GSC has more benefits from the additional paths when $L_{a}=2$.

\section{B. SHO Overhead}

In this section, we investigate the probability of the SHO attempt and the SHO overhead. In our proposed scheme, the SHO is attempted whenever $\Gamma_{L_{c}: L}$ is below $\gamma_{T}$. Hence, the probability of the $\mathrm{SHO}$ attempt is same as the outage probability of $L_{c} / L$-GSC evaluated at $\gamma_{T}$, i.e., $F_{\Gamma_{L_{c}: L}}\left(\gamma_{T}\right)$. The SHO overhead, denoted by $\beta$, is commonly used to quantify the SHO activity in a network and is defined as [17, Eq. (9.2)]

$$
\beta=\sum_{n=1}^{N} n P_{n}-1,
$$

where $N$ is the number of active BSs and $P_{n}$ is the average probability that the mobile unit uses $n$-way SHO. Based on the 
mode of operation in Section II-B, $P_{1}$ and $P_{2}$ can be defined as

$$
P_{1}=\operatorname{Pr}\left[Y+Z \geq \gamma_{T}\right]+\operatorname{Pr}\left[Y+Z<\gamma_{T}, Z \geq W\right],
$$

and

$$
P_{2}=\operatorname{Pr}\left[Y+Z<\gamma_{T}, Z<W\right] .
$$

Substituting (13) and (14) into (12), we can express the SHO overhead, $\beta$, as

$$
\begin{aligned}
\beta & =P_{1}+2 P_{2}-1 \\
& =F_{Y+Z}\left(\gamma_{T}\right) \operatorname{Pr}\left[Z<W \mid Y+Z<\gamma_{T}\right] .
\end{aligned}
$$

Note that $F_{Y+Z}\left(\gamma_{T}\right)$ is the CDF of $L_{c} / L$-GSC output SNR [16, Eq. (9.440)] evaluated at $\gamma_{T}$. Since $W$ is independent to $Z$ and $Y$, we can calculate the conditional probability, $\operatorname{Pr}\left[Z<W \mid Y+Z<\gamma_{T}\right]$, as

$$
\begin{aligned}
& \operatorname{Pr}\left[Z<W \mid Y+Z<\gamma_{T}\right] \\
& \quad=\int_{0}^{\infty} F_{Z \mid Y+Z<\gamma_{T}}(x) f_{W}(x) d x .
\end{aligned}
$$

The conditional CDF in (16) can be written as

$$
\begin{aligned}
& F_{Z \mid Y+Z<\gamma_{T}}(x) \\
& =\frac{\operatorname{Pr}\left[Z<x, Y+Z<\gamma_{T}\right]}{\operatorname{Pr}\left[Y+Z<\gamma_{T}\right]} \\
& =\frac{1}{F_{Y+Z}\left(\gamma_{T}\right)} \times \\
& \begin{cases}\int_{0}^{x} \int_{l z}^{\gamma_{T}-z}(k-l) \\
f_{Y, Z}(y, z) d y d z, & 0 \leq x<\frac{k-l}{k} \gamma_{T} ; \\
\int_{0}^{(k-l) \gamma_{T} / k} \int_{l z /(k-l)}^{\gamma_{T}-z} f_{Y, Z}(y, z) d y d z, & x \geq \frac{k-l}{k} \gamma_{T},\end{cases}
\end{aligned}
$$

where $f_{Y, Z}(y, z)$ can be obtained from (10). After successive substitutions from (17) to (15), we can finally obtain the SHO overhead. Fig. 6 shows the SHO overhead versus the output threshold, $\gamma_{T}$, of the proposed scheme for various values of $L_{a}$ over i.i.d. Rayleigh fading channels when $L=4, L_{c}=3$, and $\bar{\gamma}=0 \mathrm{~dB}$. For comparison purpose, the SHO overhead of the full GSC [13, Section IV-C] with the same parameters are plotted. Simulation results are also presented to verify our analysis. As one may expect, both schemes have the same SHO overhead when $L_{a}=1$. Overall, our proposed scheme can reduce the SHO overhead considerably. It is also very interesting to note from this figure that unlike to the full GSC scheme, our proposed scheme has a higher chance to use 2way SHO as the number of additional paths from the target BS decreases. From this figure together with Fig. 5, we can see the SHO overhead reduction of our proposed scheme. For example, for $L_{a}=2$ if the required threshold is $6 \mathrm{~dB}$ above $\bar{\gamma}$, our scheme shows around $42 \%$ of the maximum SHO overhead (in comparison with 55\% for the full GSC scheme) while achieving the minimum error rate performance for our chosen set of parameters.

\section{CONClusion}

In this paper, we proposed a new finger replacement scheme for RAKE reception in the SHO region. In this scheme, the receiver checks the GSC output SNR from the serving BS against the pre-determined output threshold. If the output SNR is below the threshold, the receiver compares the sum of the weakest paths from the serving BS with the sum of the resolvable paths from the target BS and select the better one. By deriving the joint PDF of two partial sums of order statistics, we provided an analytical framework for the statistics of the output SNR of the proposed scheme, based on which we carried out the performance analysis of the resulting systems. By investigating the tradeoff between the error performance and the SHO overhead, we showed through numerical examples that the new scheme can save the SHO overhead load considerably.

\section{REFERENCES}

[1] G. L. Stüber, Principles of Mobile Communication, 2nd ed. Norwell, MA: Kluwer Academic Publishers, 2001.

[2] T. Eng, N. Kong, and L. B. Milstein, "Comparison of diversity combining techniques for Rayleigh-fading channels," IEEE Trans. Commun., vol. 44, no. 9, pp. 1117-1129, Sept. 1996.

[3] M. Z. Win and J. H. Winters, "Analysis of hybrid selection/maximalratio combining in Rayleigh fading," IEEE Trans. Commun., vol. 47, no. 12, pp. 1773-1776, Dec. 1999.

[4] M.-S. Alouini and M. K. Simon, "An MGF-based performance analysis of generalized selection combining over Rayleigh fading channels," IEEE Trans. Commun., vol. 48, no. 3, pp. 401-415, Mar. 2000.

[5] Y. Ma and C. C. Chai, "Unified error probability analysis for generalized selection combining in Nakagami fading channels," IEEE J. Select. Areas Commun., vol. 18, no. 11, pp. 2198-2210, Nov. 2000.

[6] A. Annamalai and C. Tellambura, "Analysis of hybrid selection/maximal-ratio diversity combiner with Gaussian errors," IEEE Trans. Wireless Commun., vol. TWC-1, no. 3, pp. 498-512, July 2002.

[7] S. W. Kim, D. S. Ha, and J. H. Reed, "Minimum selection GSC and adaptive low-power RAKE combining scheme," in Proc. IEEE Int. Symp. on Circuit and Systems (ISCAS'03), Bangkok, Thailand, May 2003.

[8] H.-C. Yang, "Exact performance analysis of minimum-selection generalized selection combining (GSC)," in Proc. IEEE Int. Conf. on Commun. (ICC'05), Seoul, Korea, May 2005. Journal version to appear in IEEE Trans. Wireless Commun.

[9] R. K. Mallik, P. Gupta, and Q. T. Zhang, "Minimum selection GSC in independent Rayleigh fading," IEEE Trans. Veh. Technol., vol. 54, no. 3, pp. 1013-1021, May 2005.

[10] M.-S. Alouini and H.-C. Yang, "Minimum estimation and combining generalized selection combining (MEC-GSC)," in Proc. IEEE Int. Symp. on Information Theory (ISIT'05), Adelaide, Australia, Sept. 2005.

[11] H.-C. Yang and M.-S. Alouini, "MRC and GSC diversity combining with an output theshold," IEEE Trans. Veh. Technol., vol. 54, no. 3, pp. 1081-1090, May 2005.

[12] L. Yang and H.-C. Yang, "Performance analysis of output-threshold generalized selection combining (OT-GSC) over Rayleigh fading channels," in Proc. IEEE Wireless Comm. \& Networking Conf (WCNC'06), Las Vegas, Nevada, Apr. 2006. Journal version submitted to IEEE Trans. Wireless Commun.

[13] S. Choi, M.-S. Alouini, K. A. Qaraqe, and H.-C. Yang, "Soft handover overhead reduction by RAKE reception with finger reassignment," IEEE Trans. Commun., submitted.

[14] _ , "Performance analysis of rake receivers with finger replacement," IEEE Trans. Wireless Commun., submitted.

[15] A. Papoulis and S. U. Pillai, Probability, Random Variables, and Stochastic Processes, 4th ed. New York, NY: Mc Graw Hill, 2002.

[16] M. K. Simon and M.-S. Alouini, Digital Communication over Fading Channels, 2nd ed. New York, NY: John Wiley \& Sons, 2005.

[17] H. Holma and A. Toskala, WCDMA for UMTS, revised ed. New York, NY: John Wiley \& Sons, 2001. 


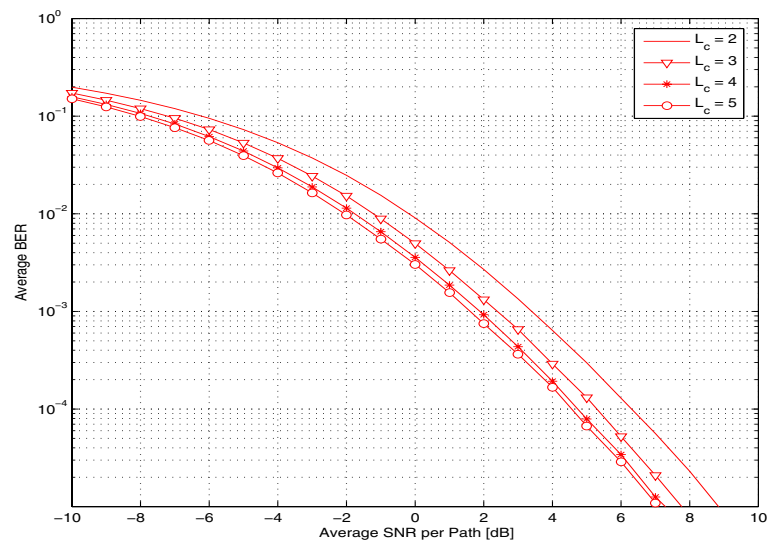

Fig. 1. Average BER of BPSK versus the average SNR per path, $\bar{\gamma}$, of the proposed scheme for various values of $L_{c}$ over i.i.d. Rayleigh fading channels when $L=5, L_{a}=1$, and $\gamma_{T}=5 \mathrm{~dB}$.

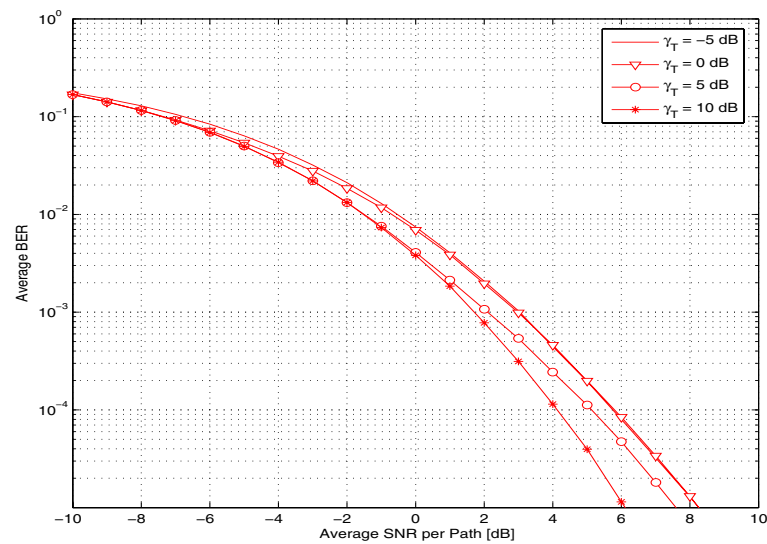

Fig. 2. Average BER of BPSK versus the average SNR per path, $\bar{\gamma}$, of the proposed scheme for various values of $\gamma_{T}$ over i.i.d. Rayleigh fading channels when $L=5, L_{c}=3$, and $L_{a}=2$.

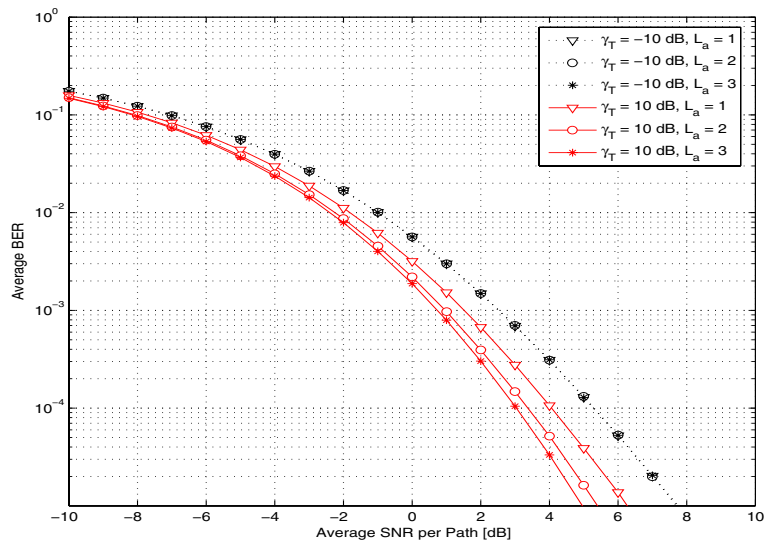

Fig. 3. Average BER of BPSK versus the average SNR per path, $\bar{\gamma}$, of the proposed scheme for various values of $L_{a}$ and $\gamma_{T}$ over i.i.d. Rayleigh fading channels when $L=5$ and $L_{c}=4$.

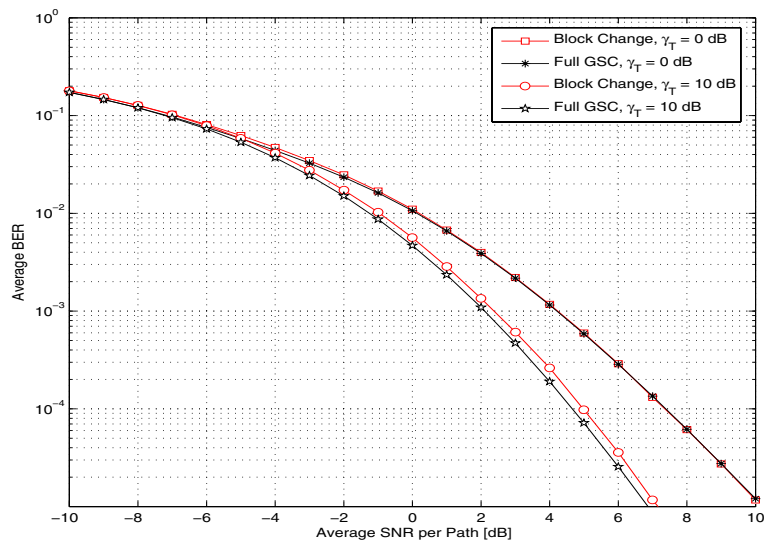

Fig. 4. Average BER of BPSK versus the average SNR per path, $\bar{\gamma}$, with the block change scheme and the full GSC scheme for various values of $\gamma_{T}$ over i.i.d. Rayleigh fading channels when $L=4, L_{c}=3$, and $L_{a}=2$.

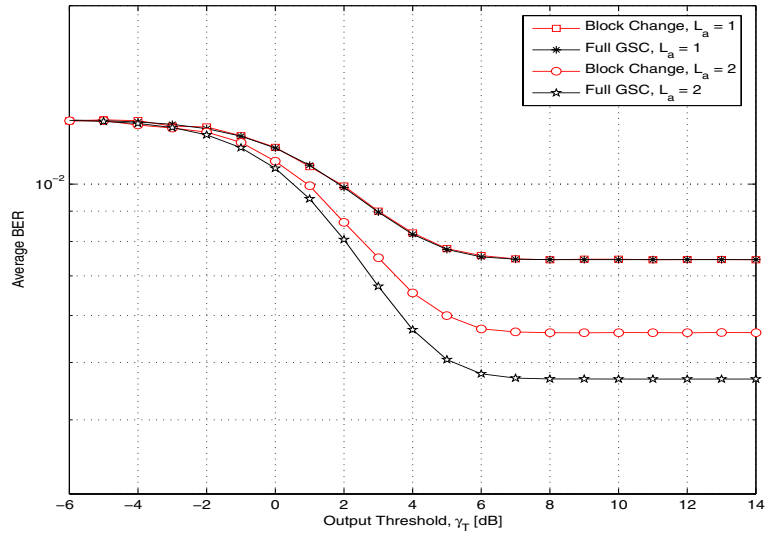

Fig. 5. Average BER of BPSK versus the output threshold, $\gamma_{T}$, with the block change scheme and the full GSC scheme for various values of $L_{a}$ over i.i.d. Rayleigh fading channels when $L=4, L_{c}=3$, and $\bar{\gamma}=0 \mathrm{~dB}$.

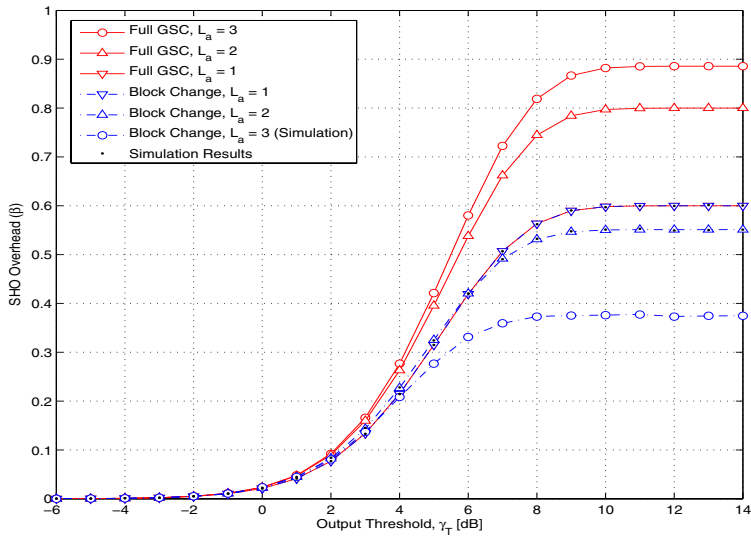

Fig. 6. SHO overhead versus the output threshold, $\gamma_{T}$, with the block change scheme and the full GSC scheme for various values of $L_{a}$ over i.i.d. Rayleigh fading channels with $L=4, L_{c}=3$, and $\bar{\gamma}=0 \mathrm{~dB}$. 